\title{
Rydberg-dressed Fermi liquid: Correlations and signatures of droplet crystallization
}

\author{
Iran Seydi $\odot,{ }^{1}$ Saeed H. Abedinpour $\odot,{ }^{1,2,3, *}$ Reza Asgari, ${ }^{4}$ Martin Panholzer $\odot,{ }^{5,6}$ and B. Tanatar ${ }^{7}$ \\ ${ }^{1}$ Department of Physics, Institute for Advanced Studies in Basic Sciences (IASBS), Zanjan 45137-66731, Iran \\ ${ }^{2}$ Research Center for Basic Sciences \& Modern Technologies (RBST), Institute for Advanced Studies in Basic Sciences (IASBS), \\ Zanjan 45137-66731, Iran \\ ${ }^{3}$ School of Nano Science, Institute for Research in Fundamental Sciences (IPM), Tehran 19395-5531, Iran \\ ${ }^{4}$ School of Physics, Institute for Research in Fundamental Sciences (IPM), Tehran 19395-5531, Iran \\ ${ }^{5}$ Institute for Theoretical Physics, Johannes Kepler University, Altenbergerstrasse 69, 4040 Linz, Austria \\ ${ }^{6}$ Uni Software Plus GmbH, 4320 Perg, Austria \\ ${ }^{7}$ Department of Physics, Bilkent University, Bilkent, 06800 Ankara, Turkey
}

(Received 1 November 2020; revised 3 March 2021; accepted 23 March 2021; published 7 April 2021)

\begin{abstract}
We investigate the effects of many-body correlations on the ground-state properties of a single-component ultracold Rydberg-dressed Fermi liquid with purely repulsive interparticle interactions in both three and two spatial dimensions. We employed the Fermi-hypernetted-chain Euler-Lagrange approximation and observed that the contribution of the correlation energy on the ground-state energy becomes significant at intermediate values of the soft-core radius and large coupling strengths. For small and large soft-core radii, the correlation energy is negligible and the ground-state energy approaches the Hartree-Fock value. The positions of the main peaks in static structure factor and pair distribution function in the homogeneous fluid phase signal the formation of quantum droplet crystals with several particles confined inside each droplet.
\end{abstract}

DOI: 10.1103/PhysRevA.103.043308

\section{INTRODUCTION}

Ultracold atoms can provide clean and controllable experimental tools to explore novel quantum phases of matter. These systems enjoy an artificial interparticle interaction that usually does not have a counterpart in conventional condensed matter systems. Rydberg atom systems, due to their long-range and strong dipole-dipole interactions, are suitable for constructing strongly correlated models and for many-body simulations [1-4]. Rydberg atoms have many applications in nonlinear quantum optics [5-7], quantum information [8-11], quantum simulation [12], and in the study of biophysical transport phenomena [13]. Optical imaging of the shape of electron orbitals of neutral atoms in Bose-Einstein condensation of Rydberg atoms has been proposed by Karpiuk et al. [14].

Usually, the lifetime of a Rydberg state is not long enough to allow the study of the atomic dynamics, but to enhance the lifetime of Rydberg atoms, the ground state could be coupled to the Rydberg state off-resonantly. In other words, with a small admixture of the Rydberg state in the ground state, it is possible to obtain long-lived Rydberg-dressed states $[2,15,16]$. Rydberg dressing for two atoms [17] and in optical lattices $[2,18]$, has been observed experimentally. The microscopy of Rydberg macro-dimers has been reported as well [19]. Rydberg-dressed atoms can be employed in the search for novel quantum phases such as the supersolid phase [15,2024], quantum liquid droplets [24-30], topological quantum magnetism [31], topological superfluidity [32], mixed topological density waves [33], and quantum spin-ice [34].

\footnotetext{
*abedinpour@iasbs.ac.ir
}

The long-anticipated supersolid phase has finally been observed in ultracold dipolar systems of magnetic atoms [35-37]. Therefore, the observation of the predicted supersolid or droplet solid phases in Rydberg-dressed systems appears very feasible.

The effect of interparticle interactions in a threedimensional (3D) Rydberg-dressed Fermi system with a pure repulsive interaction has been studied within the mean-field approximation and the density-wave instability to a metallic quantum solid phase has been predicted [38]. The groundstate properties of a two-dimensional (2D) Rydberg-dressed Fermi liquid has been investigated in the framework of the Hartree-Fock approximation and functional renormalization group $[39,40]$. The density-wave instability of the homogenous system has been reported using the random-phase approximation (RPA) [39]. With the help of the functional renormalization-group method, both $f$-wave superfluidity and density-wave instability has been predicted for Rydbergdressed fermions with repulsive interaction in 2D [40].

In this work, we address the effects of many-body correlation on the ground-state properties of a singlecomponent Rydberg-dressed Fermi liquid in both three- and two-dimensions, within the Fermi hyper-netted chain EulerLagrange (FHNC-EL) formalism at zero temperature. We show that the impact of the correlation energy on the groundstate energy becomes significant only at intermediate values of the soft-core radius and large coupling strengths. Having calculated the positions of the main peaks in the static structure factor and the pair distribution functions in the homogeneous fluid phase, we anticipate instability of the homogeneous fluid to form quantum droplet crystals with several particles confined inside each droplet. In the absence of any exact 
or state-of-the-art quantum Monte Carlo (QMC) simulation results for Rydberg-dressed fermions, we aim to verify the validity domain of the mean-field approximations as well the regimes of the system parameters where the beyond-meanfield effects become significant. The FHNC-EL formalism has been shown to provide a very accurate account of the manybody correlations in the homogeneous liquid phase [41-43], with orders of magnitude less computational demand in comparison with the QMC simulations. Furthermore, the analytic treatment of the ground state in FHNC methods allows an extension to dynamic properties [44].

The rest of this paper is organized as follows. In Sec. II, we describe our theoretical formalism and review the details of the FHNC-EL approximation. In Sec. III, we present our numerical results for different ground-state quantities of the homogeneous fluid phase such as the static structure factor, pair distribution function, effective interaction, and the ground-state energy. Furthermore, we investigate the instability of the homogeneous phase towards density-modulated phases. Finally, Sec. IV summarizes our main findings.

\section{MODEL AND THEORETICAL FORMALISM}

We consider a single-component gas of Rydberg-dressed fermions with the average density of $n$, and the bare mass of particles $m$, confined in a two- or three-dimensional space. The interaction between two Rydberg-dressed atoms is given by [15]

$$
v_{\mathrm{RD}}(r)=\frac{U}{1+\left(r / R_{c}\right)^{6}},
$$

where $R_{c}=\left[C_{6} /(2 \hbar \Delta)\right]^{1 / 6}$ is the soft-core radius of interaction and $U=[\Omega /(2 \Delta)]^{4}\left|C_{6}\right| / R_{c}^{6}$ is the interaction strength. Here $\Omega, \Delta<0$, and $C_{6}<0$ are the effective Raman coupling, red detuning and averaged van der Waals coefficient, respectively. The ground-state properties of this gas could be characterized in terms of two dimensionless parameters $u=U / \varepsilon_{\mathrm{F}}$ and $r_{c}=R_{c} k_{\mathrm{F}}$, where $\varepsilon_{\mathrm{F}}=\hbar^{2} k_{\mathrm{F}}^{2} /(2 m)$ is the Fermi energy and $k_{\mathrm{F}}=\left(2 \mathrm{~d} \pi^{\mathrm{d}-1} n\right)^{1 / \mathrm{d}}$ is the Fermi wave vector in $d$ spatial dimensions with $d=2$ or 3 .

\section{A. Fermi-hypernetted-chain Euler-Lagrange approximation}

Taking the chemical potential of the system as the zero point of energy, we can write the differential equation for the pair distribution function $g(r)[41,42]$ as

$$
\left[-\frac{\hbar^{2}}{m} \nabla_{\mathbf{r}}^{2}+w_{\text {eff }}(r)\right] \sqrt{g(r)}=0,
$$

where $w_{\text {eff }}(r)$ is the effective potential and in practice needs to be approximated. Unlike the bosonic systems, a truly FHNC formulation for the effective interaction in Fermi gases leads to a very complicated set of coupled equations [45]. However, several simplified recipes have tried to implement the exact weak or strong-coupling limiting behavior in the effective interaction and proved to give reasonably accurate results in the corresponding limits $[46,47]$. In this work, we follow the recipe of Kallio and Piilo (KP), which has been tailored to exactly capture the Fermi statistics and weak-coupling behavior [46]. For an alternative approximation, based on the approximate summation of ladder and ring diagrams, see Appendix B.

Within the KP approximation the effective interaction is given by

$$
w_{\mathrm{eff}}(r)=v_{\mathrm{RD}}(r)+w_{\mathrm{B}}(r)+w_{\mathrm{F}}(r),
$$

where the bosonic potential $w_{\mathrm{B}}(q)$ in the Fourier space, at the level of HNC-EL/0 approximation, i.e., neglecting the elementary diagrams and correlations higher than pair correlations, is given by

$$
w_{\mathrm{B}}(q)=-\frac{\varepsilon_{q}}{2 n}[2 S(q)+1]\left[\frac{S(q)-1}{S(q)}\right]^{2} .
$$

Here, $\varepsilon_{q}=\hbar^{2} q^{2} /(2 m)$ is the noninteracting dispersion and $S(q)$ is the static structure factor, related to the pair distribution function as $S(q)=1+n \mathcal{T}_{F}[g(r)-1]$, where the Fourier transform $\mathcal{T}_{F}$ is defined as $\int d \mathbf{r} f(r) e^{-i \mathbf{q} \cdot \mathbf{r}}$. The Fermi contribution to the effective potential $w_{\mathrm{F}}(r)$, which includes most importantly the exchange effects, within the KP approximation reads

$$
w_{\mathrm{F}}(r)=\frac{\hbar^{2}}{m} \frac{\nabla_{\mathbf{r}}^{2} \sqrt{g_{0}(r)}}{\sqrt{g_{0}(r)}}-\lim _{u \rightarrow 0} w_{\mathrm{B}}(r),
$$

where $g_{0}(r)$ is the pair distribution function of a noninteracting Fermi gas [48] and the noninteracting limit of the Bose potential could be obtained after replacing the static structure factor in Eq. (4) with $S_{0}(q)$, the static structure factor of a noninteracting Fermi gas [48].

A numerically efficient strategy to solve the zero-energy differential equation (2) is to invert it and obtain the effective potential

$$
V(r)=g(r) w_{\text {eff }}(r)-w_{\mathrm{B}}(r)+\frac{\hbar^{2}}{m}|\nabla \sqrt{g(r)}|^{2},
$$

whose Fourier transform gives the static structure factor

$$
S(q)=\frac{1}{\sqrt{1+2 n V(q) / \epsilon_{q}}} .
$$

Now, Eqs. (4), (6), and (7) could be solved self-consistently for a given set of system parameters $u$ and $r_{c}$. Note that the Fermi potential in the KP formalism, as given by Eq. (5), is already fixed by the noninteracting pair distribution function and structure factor, and does not enter the loop of self-consistency.

\section{B. The ground-state energy}

Once the pair distribution function is known for different interaction strengths, the ground-state energy per particle could be obtained from the coupling constant integration [48]

$$
\varepsilon_{\mathrm{GS}}\left(u, r_{c}\right)=\varepsilon_{0}+\frac{n}{2} \int_{0}^{u} d u^{\prime} \int d \mathbf{r} \frac{g_{u^{\prime}}(r)}{1+\left(r / R_{c}\right)^{6}} .
$$

Here, $\varepsilon_{0}=\varepsilon_{\mathrm{F}} d /(d+2)$ is the noninteracting kinetic energy of a $d$-dimensional Fermi gas and $g_{u^{\prime}}(r)$ is the interacting pair distribution function of a Rydberg-dressed Fermi liquid with interaction strength equal to $u^{\prime}$ and at fixed soft core radius $r_{c}$. The correlation energy, which is defined as the difference between the exact ground-state and the restricted 
Hartree-Fock energies is a good measure of the performance of any approximate theories. In the next section, we report our numerical results for the ground-state and correlation energies as functions of the interaction strength $u$ and the soft-core radius $r_{c}$.

\section{Density-density response function}

The collective density modes and signatures of the instability of a homogeneous system to density modulated phases both could be obtained from the singularities of its densitydensity response function

$$
\chi(q, \omega)=\frac{\chi_{0}(q, \omega)}{1-V_{\mathrm{ph}}(q, \omega) \chi_{0}(q, \omega)},
$$

respectively in the dynamic and static regimes. Here, $\chi_{0}(q, \omega)$ is the noninteracting density-density response function [48], and $V_{\mathrm{ph}}(q, \omega)$ is the particle-hole irreducible interaction [44], which needs to be approximated for any practical purpose. In the acclaimed random-phase approximation (RPA), all the exchange and correlation effects are discarded, replacing the effective interaction with the bare interaction. Schemes to go beyond the RPA mainly rely on introducing the many-body local-field factors [48]. On the other hand, if the interacting static structure factor is known, the fluctuation-dissipation theorem

$$
S(q)=-\frac{\hbar}{n \pi} \int_{0}^{\infty} d \omega \operatorname{Im} m[\chi(q, \omega)],
$$

could be used to extract a static effective interaction [43]. Further approximating the noninteracting density-density response function of the Fermi gas with a Bose-like expression, i.e., the "mean spherical approximation" (MSA)

$$
\chi_{0}^{\mathrm{MSA}}(q, \omega)=\frac{2 n \varepsilon_{q}}{\left(\hbar \omega+i 0^{+}\right)^{2}-\left[\varepsilon_{q} / S_{0}(q)\right]^{2}},
$$

the frequency integral in Eq. (10) could be performed analytically and a simple analytic expression for the static effective interaction is obtained:

$$
V_{\mathrm{ph}}(q)=\frac{\varepsilon_{q}}{2 n}\left[\frac{1}{S^{2}(q)}-\frac{1}{S_{0}^{2}(q)}\right] .
$$

In this work, we use the static structure factor obtained from the solution of KP equations to extract the static effective interaction. This approach has proved to give very good results for different properties of various strongly interacting Fermi liquids $[49,50]$.

\section{NUMERICAL RESULTS AND DISCUSSION}

In this section, we turn to the presentation of our numerical results for static structure factor, pair distribution function, and effective interaction $w_{\text {eff }}(r)$ of a one-component Rydbergdressed Fermi liquid obtained from the KP approximation. We also investigate the contribution of correlation energy to the total ground-state energy at different system parameters. Finally, we discuss the dynamical structure factor and the density-wave instability of the homogeneous Rydbergdressed Fermi liquid.
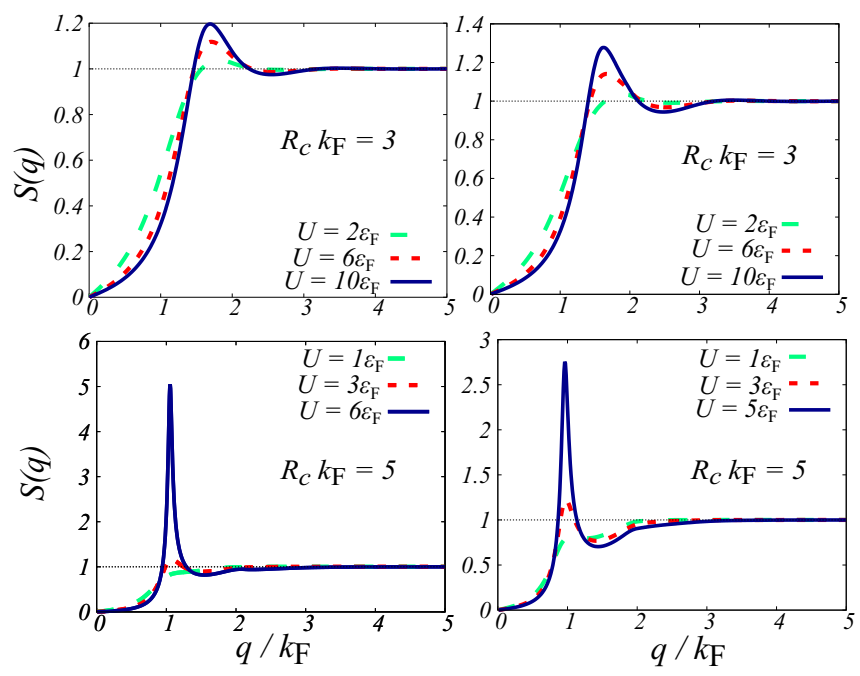

FIG. 1. The static structure factor of 3D (left) and 2D (right) Rydberg-dressed Fermi liquid versus $q / k_{\mathrm{F}}$ obtained from the solution of KP equations at two fixed values of the dimensionless soft-core radius $r_{c}=R_{c} k_{\mathrm{F}}$ and for different values of the interaction strength $u=U / \varepsilon_{\mathrm{F}}$.

\section{A. Static structure factor}

Figure 1 shows the static structure factors of three- and two-dimensional Rydberg-dressed Fermi liquids, obtained from the solution of KP equations at different values of the soft-core radius $r_{c}$ and coupling constant $u$. For a fixed value of the soft-core radius, increasing the interaction strength, the height of the main peak in $S(q)$ increases, indicating the enhancement of correlations. Another interesting observation in the behavior of the static structure factor is the location of its main peak. The position of the main peak of $S(k)$ in the homogeneous state predicts the Bragg peak of the crystalline phase. Our numerical results show that this peak moves to smaller wave vectors as the correlations get stronger. This is an indication of the tendency of the system to the formation of crystalline structures with a larger lattice constant in the ordered phase, which means having a larger number of atoms in each unit cell.

\section{B. Pair distribution function and effective interaction}

The pair distribution function $g(r)$ gives the relative spatial distribution of particles in the system, therefore is a positivedefinite function. The pair distribution function is normalized such that $\lim _{r \rightarrow \infty} g(r) \rightarrow 1$, since the correlation between particles vanishes at large separations.

Figure 2 illustrates the pair distribution function of Rydberg-dressed Fermi liquids at different values of the softcore radius $r_{c}$ and coupling constant $u$. It is interesting to note that the positivity of the pair distribution function and the exact condition $g(r=0)=0$ for our spin-polarized Fermi system, as implied by Pauli's exclusion principle, are both satisfied within the approximate KP formalism, even up to very strong interaction strengths. Notice that the vanishing on top value of the pair distribution function for classical particles originates from their impenetrability. In our model 

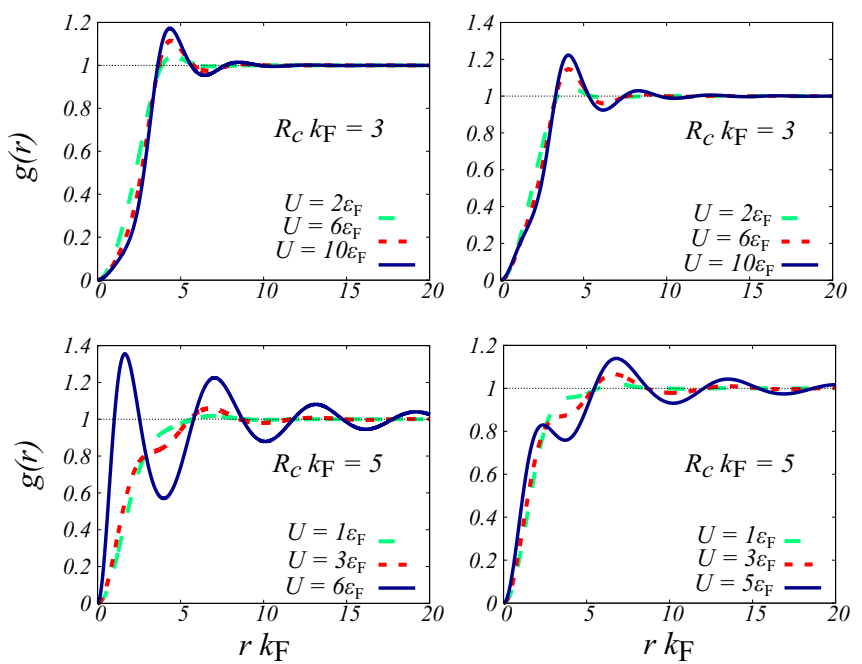

FIG. 2. The pair distribution function versus $r k_{\mathrm{F}}$ within the KP approximation at two fixed values of the soft-core radius and for different values of the interaction strength for 3D (left) and 2D (right) Rydberg-dressed Fermi liquids.

of point particles with soft repulsive interaction, this is simply due to Pauli's exclusion principle.

Apart from the well-expected behavior of the pair distribution function of the liquid phase at strong correlations, i.e., a pronounced first peak at a specific distance and slowly decaying oscillations, here an interesting observation is the appearance of a shoulder at small distances which evolves into a peak and eventually dominates the original first-peak of the pair distribution function at large soft-core radius and strong couplings. This indicates a smaller first-neighbor distance than the average interparticle separation or the tendency of particles to aggregate at strong interactions. This is quite counterintuitive, keeping in mind the repulsive nature of the bare interparticle interaction. When considered together with the peculiar behavior of the main peak in the static structure factor, this could be an indication of the tendency of the system for the formation of quantum droplet crystals at strong correlations. The main peak position of $S(k)$ moves to smaller wave vectors as the correlation increases, indicating a larger lattice constant in the ordered phase. At the same time, the first neighbor distance in the pair distribution function gets smaller due to the clustering of several particles inside each droplet. As a result, the average distance between ordered droplets becomes larger and the number of atoms in each droplet increases (see Table I).

The above-mentioned distinctive behavior of the pair distribution function could be understood from the effective interaction $w_{\text {eff }}(r)$, as illustrated in Fig. 3. Apart from the repulsive hard-core of the effective interaction which originates from the statistical i.e., Pauli repulsion, the effective interaction becomes attractive around the distance where the first peak of the pair distribution function appears. At strong couplings, the effective interaction has an oscillatory behavior, and its first minimum moves towards smaller distances, in agreement with the behavior of the pair distribution function. It is worth mentioning that quantum fluctuations are enhanced in $2 \mathrm{D}$ and the possibility of observing droplets in $2 \mathrm{D}$ is of
TABLE I. Instability wave vector $q_{I}$ (in units of $k_{\mathrm{F}}$ ), lattice constant $a$ (in units of $1 / k_{\mathrm{F}}$ ), and the rounded number of particles per droplet $N_{d}$, of Rydberg-dressed fermions in a body-centered cubic three-dimensional and a two-dimensional triangular lattice structure for several values of the soft-core radius $r_{c}$ and interaction strength $u$ in the vicinity of the density-wave instability.

\begin{tabular}{llllrr}
\hline \hline & $r_{c}$ & $u$ & $q_{I}\left[k_{\mathrm{F}}\right]$ & $a\left[1 / k_{\mathrm{F}}\right]$ & $N_{d}$ \\
\hline 3D & 4 & 9 & 1.28 & 6.94 & 3 \\
3D & 5 & 6 & 1.055 & 8.42 & 5 \\
3D & 6 & 2 & 0.89 & 9.98 & 8 \\
3D & 7 & 1 & 0.77 & 11.54 & 13 \\
2D & 4 & 7 & 1.22 & 5.95 & 2 \\
2D & 5 & 5 & 0.97 & 7.48 & 4 \\
2D & 6 & 3 & 0.81 & 8.96 & 6 \\
2D & 7 & 2 & 0.69 & 10.51 & 8 \\
\hline \hline
\end{tabular}

great interest and, as shown in Fig. 3, attractive effective potential permits the formation of self-bound quantum droplets.

\section{The ground-state and correlation energies}

The ground-state energy per particle of the Rydbergdressed Fermi liquid could be written in terms of different contributions to it:

$$
\varepsilon_{\mathrm{GS}}\left(u, r_{c}\right)=\varepsilon_{0}+\varepsilon_{\mathrm{H}}\left(u, r_{c}\right)+\varepsilon_{x}\left(u, r_{c}\right)+\varepsilon_{c}\left(u, r_{c}\right),
$$

where $\varepsilon_{0}=\varepsilon_{\mathrm{F}} d /(d+2)$ is the noninteracting kinetic energy of a $d$-dimensional Fermi gas and the Hartree energy is given by

$$
\varepsilon_{\mathrm{H}}\left(u, r_{c}\right)=\frac{n}{2} v_{\mathrm{RD}}(q=0)=\alpha_{d} u r_{c}^{d} \varepsilon_{\mathrm{F}}
$$

with $\alpha_{d}=(\pi / \sqrt{3})^{3-d} /(6 d)$. The exchange energy per particle could be obtained from [48]

$$
\varepsilon_{x}\left(u, r_{c}\right)=-\frac{1}{2 N L^{d}} \sum_{\mathbf{q}} v_{\mathrm{RD}}(q) \sum_{\mathbf{k}} n_{\mathbf{k}+\mathbf{q}} n_{\mathbf{k}},
$$
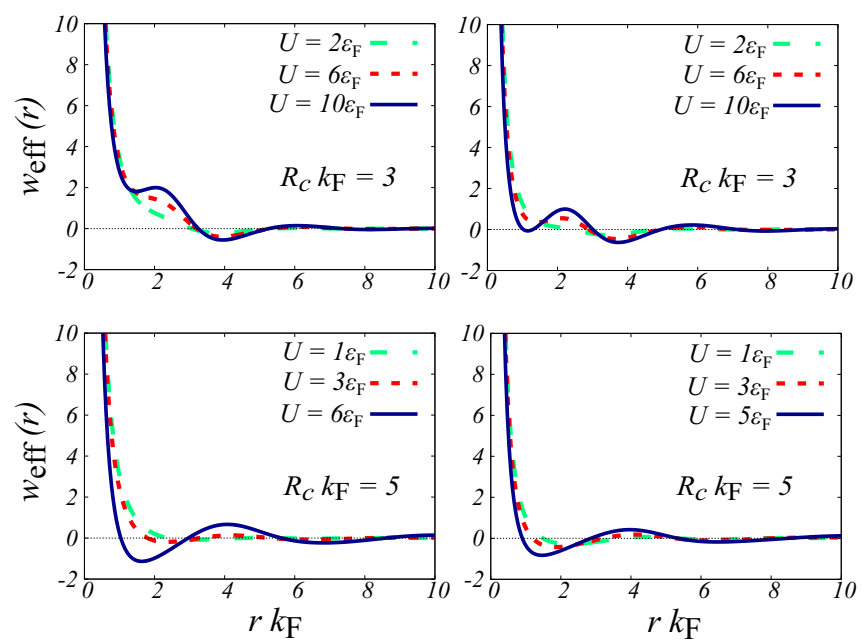

FIG. 3. The effective interaction $w_{\text {eff }}(r)$ (in units of the Fermi energy $\varepsilon_{\mathrm{F}}$ ) versus $r k_{\mathrm{F}}$ obtained within the $\mathrm{KP}$ approximation at two fixed values of $R_{c} k_{\mathrm{F}}$ and for different values of the interaction strength for 3D (left) and 2D (right) Rydberg-dressed Fermi liquids. 

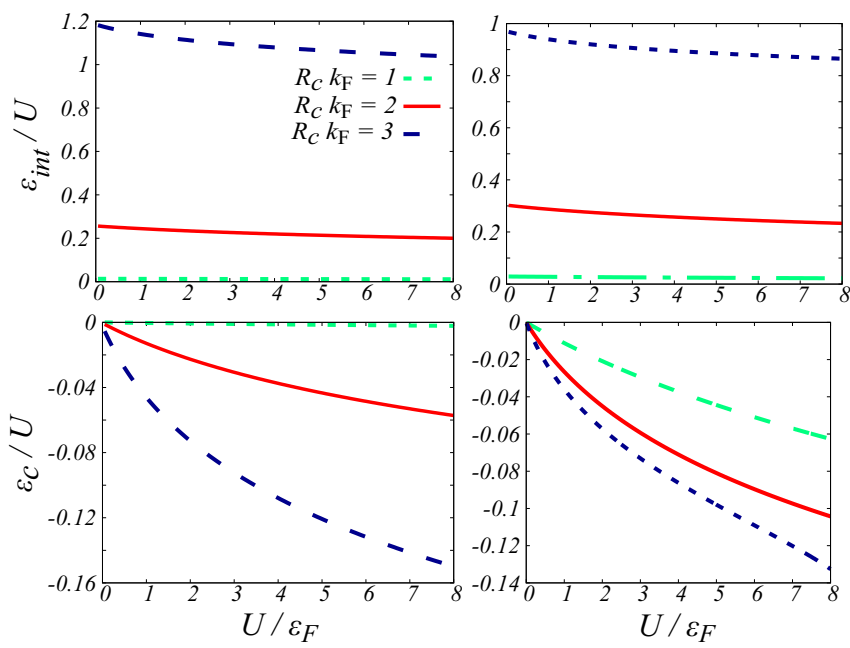

FIG. 4. (top panels) The interacting part of the ground-state energy per particle $\varepsilon_{\text {int }}$ (in units of the interaction strength $U$ ) versus $U / \varepsilon_{\mathrm{F}}$ for different values of the soft-core radius, obtained from the KP approximation for 3D (left) and 2D (right) Rydberg-dressed Fermi liquids. (bottom panels) Same as the top panels, but for the correlation energy $\varepsilon_{c}$.

where $N$ is the particle number, $L$ is the sample length, and $n_{\mathbf{k}}$ is the Fermi-Dirac distribution function, which becomes a step function $\Theta\left(\varepsilon_{\mathrm{F}}-\varepsilon_{k}\right)$ at zero temperature. Analytic summation over $\mathbf{q}$ and $\mathbf{k}$ in Eq. (15) is possible and yields

$$
\begin{aligned}
\varepsilon_{x}^{(3 \mathrm{D})}\left(u, r_{c}\right)= & \frac{\varepsilon_{\mathrm{F}} u}{4 r_{c}^{3}}\left\{e^{-2 r_{c}}\left(1+r_{c}\right)^{2}-2 r_{c}^{3}+2 r_{c}^{2}+1\right. \\
& -e^{-r_{c}}\left[\sqrt{3}\left(2 r_{c}+r_{c}^{2}\right) \sin \left(\sqrt{3} r_{c}\right)\right. \\
& \left.\left.+\left(2+2 r_{c}-r_{c}^{2}\right) \cos \left(\sqrt{3} r_{c}\right)\right]\right\},
\end{aligned}
$$

and [39]

$$
\varepsilon_{x}^{(2 \mathrm{D})}\left(u, r_{c}\right)=\frac{\varepsilon_{\mathrm{F}} u}{2}\left[1-\frac{G_{3,9}^{4,3}\left(\begin{array}{l|l|l|}
r_{c}^{6} & \frac{1}{6}, \frac{1}{2}, \frac{5}{6} \\
0, \frac{1}{3}, 1,-\frac{1}{3}, \frac{1}{3}, \frac{2}{3}
\end{array}\right)}{3 \sqrt{3}}\right],
$$

in three and two dimensions, respectively. Here, $G(\cdots)$ is the Meier $G$ function [51].

The correlation energy $\varepsilon_{c}$ itself is defined through Eq. (13) as the difference between the exact ground-state energy and the Hartree-Fock energy and needs to be approximated. We use the coupling constant integration as introduced through Eq. (8), with the pair distribution function obtained from the KP calculations to find the correlation energy of three- and two-dimensional Rydberg-dressed Fermi liquids for different values of the coupling strengths and soft-core radii.

In Fig. 4 we present the interaction contribution to the ground-state energy $\varepsilon_{\text {int }}=\varepsilon_{\mathrm{GS}}-\varepsilon_{0}$ and the correlation energy $\varepsilon_{c}$ versus the interaction strength for different values of the soft-core radius. The correlation energy, as expected, is negative but the total ground-state energy is an increasing function of both $u$ and $r_{c}$. As it is clear from the analytic expressions (14) and (15), very weak nonlinear $u$ dependence of the interaction energy (top panels of Fig. 4) originates solely from the correlation energy (bottom panels of Fig. 4).

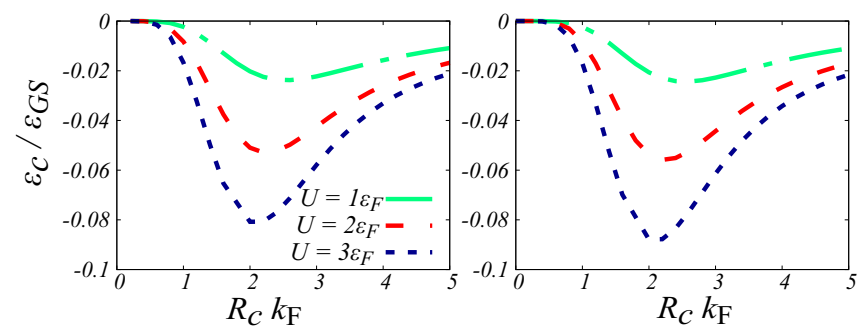

FIG. 5. The correlation energy per particle $\varepsilon_{c}$ in units of the total ground-state energy $\varepsilon_{\mathrm{GS}}$, as a function of the soft-core radius $r_{c}$ at several values of the coupling strength $u$, calculated within the $\mathrm{KP}$ formalism for 3D (left) and 2D (right) Rydberg-dressed Fermi liquids.

Figure 5 illustrates the soft-core radius dependence of the correlation energy of a Rydberg-dressed Fermi liquid. The correlation energy has a considerable contribution to the ground-state energy only at intermediate values of the softcore radii (i.e., $r_{c} \approx 2$ ) and at large coupling strengths. For both small and large values of $r_{c}$, the correlation energy has a small value, and the KP ground-state energies approach the mean-field HF results. This can be attributed to the behavior of the bare potential. At small values of $R_{c}$, the potential is short-ranged decaying as $1 / r^{6}$, and for large $R_{c}$ the potential is almost constant.

\section{The dynamical structure factor and collective modes}

The zero-temperature dynamical structure factor is proportional to the imaginary part of the interacting density-density response function

$$
S(q, \omega)=-\frac{\hbar}{n \pi} \operatorname{Im} m \chi(q, \omega) .
$$

Using a static approximation for the effective interaction, such as the one given by Eq. (12), the imaginary part of the densitydensity response function remains nonzero only inside the single-particle excitation continuum, where the imaginary part of the noninteracting density-density response function is nonzero and along the dispersion of collective density mode, where it is proportional to a Dirac delta peak [48]. In Fig. 6 we illustrate the dynamical structure factor for three- and two-dimensional Rydberg-dressed Fermi liquids at a fixed interaction strength and for two different values of the softcore radius. The broadening of the collective mode inside the single-particle excitation continuum due to its damping into particle-hole pairs is evident. At larger values of the soft-core radius (bottom panels) softening of the collective mode inside the continuum is an indication of the density-wave instability, which will be discussed in detail in the next section.

\section{E. Density-wave instability}

When the static density response function of a homogeneous system diverges, or equivalently its static dielectric function becomes zero at a specific wave vector $q_{I}$, the homogeneous system becomes unstable to a density-modulated phase with wavelength $\lambda_{I}=2 \pi / q_{I}$. However, one should note that this instability corresponds to a second-order phase 

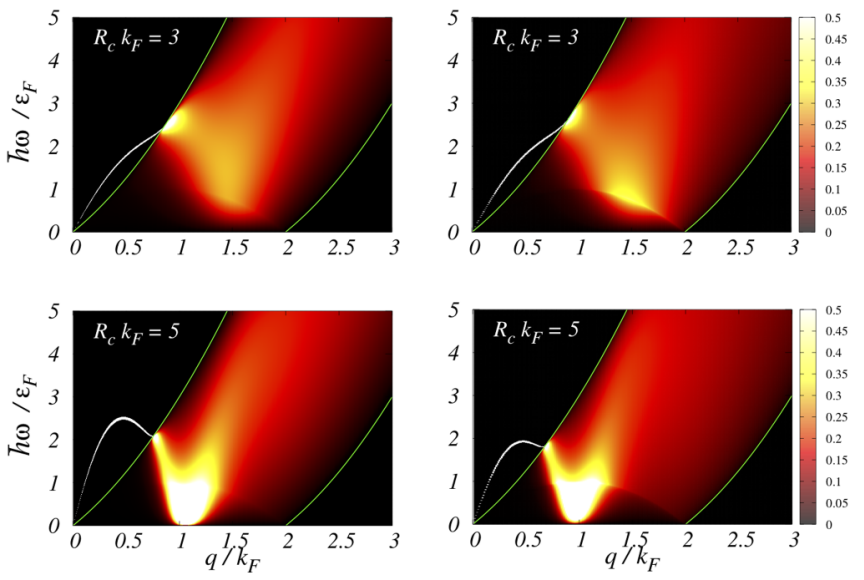

FIG. 6. The density plots of the dynamical structure factor (in units of $\hbar / \varepsilon_{\mathrm{F}}$ ) versus $q / k_{\mathrm{F}}$ and $\hbar \omega / \varepsilon_{\mathrm{F}}$ at a fixed value of the interaction strength $U=3 \varepsilon_{\mathrm{F}}$ and for two fixed values of the soft-core radius for 3D (left) and 2D (right) Rydberg-dressed Fermi liquids. The green lines $\hbar^{2} q^{2} /(2 m) \pm \hbar^{2} k_{\mathrm{F}} q / m$, show the borders of the single-particle excitation continuum where the imaginary part of the noninteracting density-density response function is nonzero. The imaginary part of the density-density response function outside the continuum is broadened by $10^{-4}$ to make the Dirac delta peak of the collective mode visible.

transition in the density channel and relies on the presumption that no first-order phase transition, or a competing secondorder phase transition in other channels, precedes it.

Using the mean-field Hartree-Fock approximation and the above-mentioned density instability criterion from the density response function within the RPA, a metallic quantum solid phase has been predicted for a 3D system of Rydberg-dressed fermions [38]. The mean-field method predicted a first-order phase transition from a homogeneous phase to the bcc crystalline structure, but interestingly the phase boundary between liquid and solid phases obtained from two techniques was in very good agreement. For a two-dimensional system of Rydberg-dressed fermions, density instability has been investigated using the RPA for the density-density response function [39] too. In Fig. 7, we compare the phase diagram of Rydberg-dressed fermions obtained from the poles of the density-density response function (9) in the static limit, within the RPA, where $V_{\mathrm{ph}}(q)$ is replaced with the bare interaction $v_{\mathrm{RD}}(q)$, and the FHNC approximation, where the effective interaction is extracted from Eq. (12) using the numerical data for the static structure factor obtained from KP calculations. In the latter approximation, the effects of the exchange and correlation holes are approximately included in the effective interaction. This inclusion of exchange-correlation effects within the KP approximation makes the homogeneous liquid phase more stable. This is in line with the results obtained from the improvements over RPA with the inclusion of the Hubbard local-field factor [39]. However, the phase boundary seems not to be very sensitive to the exchange-correlation at intermediate and large soft core radii. At small values of $r_{c}$, the difference between the two approximations becomes significant and, eventually, we do not find any instability at

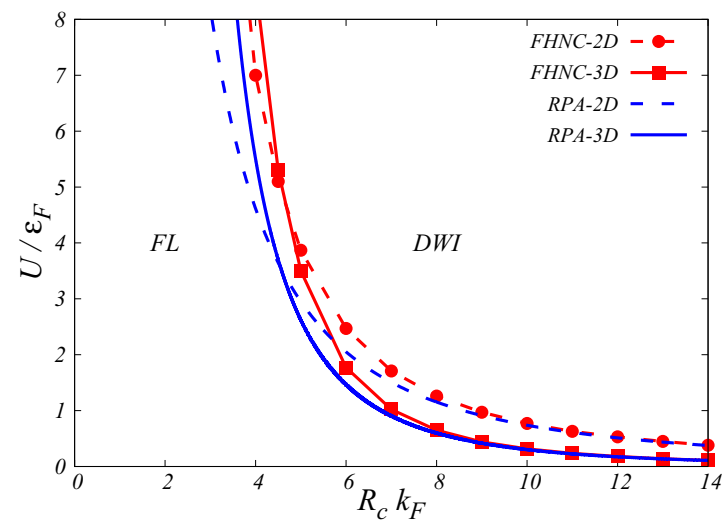

FIG. 7. The phase diagram of 3D and 2D Rydberg dressed fermions versus $u$ and $r_{c}$, obtained from the RPA and FHNC approximation. The stable homogeneous Fermi liquid (FL) phase and regions where it becomes unstable towards the density wave instability phase (DWI) are shown in the phase diagram.

much smaller soft-core radii when we include the effects of exchange-correlation in the effective interaction.

As the static noninteracting density-density response function $\chi_{0}(q, \omega=0)$ is always negative, the vanishing of the denominator of Eq. (9) is possible only in the regions of wave vector where the effective interaction is also negative. As the Fourier transform of the bare interaction $v_{\mathrm{RD}}(q)$ becomes negative around $q \approx 5 / R_{c}$, the wavelength of the density-modulated phase would be directly proportional to the soft-core radius within the RPA. At small enough soft-core radii, the RPA unphysically predicts instability whose wavelength $\lambda_{I}$ is much smaller than the average distance between particles, i.e., $q_{I} \gg 2 k_{\mathrm{F}}$ [39]. In contrast, when the effective interaction is extracted from the static structure factor, the instability wave vector is given by the location of the main peak in $S(q)$. As discussed in Sec. III B, this is related to the average spacing between droplets in the crystalline phase, and never exceeds $2 k_{\mathrm{F}}$.

To obtain the lattice constant and the approximate number of atoms in each droplet, we determine the instability wave vector from the main peak of the static structure factor and then obtain the lattice constant and the number of atoms in each droplet. The wave vector corresponding to the main peak of the static structure factor is related to the lattice constant through $q_{I}=(2 \pi \sqrt{2}) / a_{\mathrm{bcc}}$ and $q_{I}=4 \pi /\left(\sqrt{3} a_{\mathrm{tri}}\right)$, in three-dimensional body-centered cubic and two-dimensional triangular lattices, respectively. The number of atoms in each droplet is given by $N_{d}=n \Omega$, where $n$ is the density of particles which is given in terms of the Fermi wave vector as $n^{(3 \mathrm{D})}=k_{\mathrm{F}}^{3} /\left(6 \pi^{2}\right)$ and $n^{(2 \mathrm{D})}=k_{\mathrm{F}}^{2} /(4 \pi)$. The volume (or area) of the unit cell $\Omega$ is related to the lattice constant as $\Omega^{(3 \mathrm{D})}=a_{\mathrm{bcc}}^{3} / 2$ and $\Omega^{(2 \mathrm{D})}=\sqrt{3} a_{\mathrm{tri}}^{2} / 2$, respectively, for the chosen three- and two-dimensional lattice structures.

We have reported the properties of representative lattice structures made of quantum droplets, in three and two dimensions, in Table I. In both spatial dimensions, increasing the dimensionless soft-core radius $r_{c}$, the lattice constant and the number of particles in each droplet increases. 


\section{SUMMARY}

We have studied the ground-state properties of Rydbergdressed Fermi liquids in two and three dimensions in the framework of Fermi-hypernetted-chain Euler-Lagrange approximation. The emergence of an extra small distance peak in the pair distribution function and the shift of the main peak in the static structure factor to long wavelengths with increasing interaction strength or the soft-core radius signals the phase transition from the homogenous Fermi liquid to quantum droplet crystalline phase.

We expect that our anticipated quantum droplet regime to be experimentally accessible. If one considers ${ }^{40} \mathrm{~K}$ atoms excited to the $62 \mathrm{~S}$ state, the bare van der Waals coefficient would be $C_{6} / \hbar \approx-2 \pi \times 129.8 \mathrm{GHz} \mu \mathrm{m}^{6}$ [52]. The soft core radius of $R_{c} \approx 5.6 \mu \mathrm{m}$ is obtained for the red laser detuning frequency of $|\Delta| \approx 2 \pi \times 2 \mathrm{MHz} . r_{c} \approx 5$ requires $n^{3 \mathrm{D}} \approx$ $1.2 \times 10^{10} \mathrm{~cm}^{-3}$ and $n^{2 \mathrm{D}} \approx 6.2 \times 10^{6} \mathrm{~cm}^{-2}$ in three and two dimensions, respectively, and the Rabi frequency of at least $\Omega \approx 2 \pi \times 400 \mathrm{kHz}$ would be necessary to observe the formation of quantum droplets (i.e., to get $u \approx 4$ ).

We have also calculated the ground-state energy of the homogeneous liquid phase. We found that the correlation energy is considerable only at intermediate values of the soft-core radii. At both small and large values of the soft-core parameter, the mean-field approximation seems to be adequate to describe the physics of the Rydberg-dressed fermions. We should note that, to the best of our knowledge, more accurate numerical techniques such as the quantum Monte Carlo simulations are not yet available for Rydberg-dressed fermions. However, previous experience with Fermi liquids with other forms of interactions [41-43], suggests that the KP results for the ground-state energy are generally reliable up to very strong couplings. The relative error in comparison to the exact results is not expected to exceed a few percent.

Our results for the correlation energy of the homogeneous Rydberg-dressed Fermi liquid could be used as input for the density-functional theory like studies of inhomogeneous systems, such as the more realistic trapped systems.

We should also note that we have examined other approximate methods such as the RPA and Singwi-Tosi-LandSjölander (STLS) approximations [53] to find the groundstate properties and the correlation energy of Rydberg-dresses Fermi liquids [54]. Notably, within the RPA the correlation energy is strongly overestimated. This leads to an erroneous prediction of the self-bound state for Rydberg-dressed fermions. The STLS approximation, although it largely improves the RPA results for the ground-state energy, even at intermediate couplings its pair distribution function severely violates the exact conditions, i.e., the positivity and vanishing on-top value [54].

\section{ACKNOWLEDGMENTS}

We thank Robert E. Zillich, Eugene Demler, and Andy Schofield for very fruitful discussions. S.H.A. is supported by Iran Science Elites Federation (ISEF). B.T. acknowledges support from The Scientific and Technological Research Council of Turkey (TUBITAK) and Turkish Academy of Sciences (TUBA).

\section{APPENDIX A: HYPERNETTED-CHAIN EULER-LAGRANGE METHOD}

Here, we provide a brief overview of the microscopic description of a homogeneous many-body quantum system at zero temperature and in the thermodynamic limit, within the HNC-EL formalism. We consider the following Hamiltonian for the interacting system:

$$
H=\sum_{i=1}^{N} \frac{p_{i}^{2}}{2 m}+\sum_{i<j}^{N} v\left(r_{i j}\right) .
$$

Whenever interactions between particles become strong, perturbation theory is no longer applicable to solve the manyparticle Schrödinger equation. Therefore, a nonperturbative approach such as HNC-EL variational approach is needed. Within this approach, the ground-state wave function is approximated as

$$
\Psi_{T}=F_{N}(1, \ldots, N) \phi(1, \ldots, N),
$$

where $F_{N}(1, \ldots, N)$ is the $N$-particle correlation operator that is assumed to be symmetric and $\phi(1, \ldots, N)$ is a wave function of a noninteracting model of the system and determines the symmetry of the ground-state wave function. Discarding the three-body and higher-order correlations, the correlation operator assumes the Jastrow-Feenberg form given by

$$
F\left(\mathbf{r}_{1}, \ldots, \mathbf{r}_{N}\right)=\prod_{i<j}^{N} f\left(r_{i j}\right),
$$

where $f(r)$ is the Jastrow factor and it goes to zero as $r \rightarrow 0$ and tends to unity as $r \rightarrow \infty$. The exact form of $f(r)$ is not known, but it can be determined by variational method

$$
\frac{\partial}{\partial f} \frac{\left\langle\Psi_{T}|\hat{H}| \Psi_{T}\right\rangle}{\left\langle\Psi_{T} \mid \Psi_{T}\right\rangle}=0 .
$$

The exact solution of this equation could be obtained, e.g., from Monte Carlo methods, but here we use the analytic cluster expansion method to calculate the integrals in the expectation value of Hamiltonian [55]. The contribution of potential energy in the expectation value of Hamiltonian is calculated as follows:

$$
\begin{aligned}
\frac{1}{N}\langle\hat{V}\rangle & \equiv \frac{1}{N} \frac{\left\langle\Psi_{T}\left|\sum_{i<j} v\left(r_{i j}\right)\right| \Psi_{T}\right\rangle}{\left\langle\Psi_{T} \mid \Psi_{T}\right\rangle} \\
& =\frac{N(N-1)}{2 N} \frac{\left\langle\Psi_{T}\left|v\left(r_{12}\right)\right| \Psi_{T}\right\rangle}{\left\langle\Psi_{T} \mid \Psi_{T}\right\rangle} \\
& =\frac{n^{2}}{2 N} \int d \mathbf{r}_{1} d \mathbf{r}_{2} v\left(r_{12}\right) g\left(r_{1}, r_{2}\right),
\end{aligned}
$$

where the two-particle radial distribution function is defined as

$$
g\left(r_{1}, r_{2}\right) \equiv \frac{N(N-1)}{n^{2}} \frac{\int d \mathbf{R}_{12}\left|\Psi_{T}\right|^{2}}{\int d \mathbf{R}\left|\Psi_{T}\right|^{2}},
$$

with $\int d \mathbf{R}=\int d \mathbf{r}_{1} \ldots d \mathbf{r}_{N}$ and $\int d \mathbf{R}_{12}=\int d \mathbf{r}_{3} \ldots d \mathbf{r}_{N}$. For uniform and homogeneous systems that are invariant under rotation and translation, $g\left(r_{1}, r_{2}\right)$ depends only on $r=r_{1}-r_{2}$, 
and we find

$$
\frac{1}{N}\langle\hat{V}\rangle=\frac{n}{2} \int d \mathbf{r} v(r) g(r)
$$

Obtaining the contribution of the kinetic energy is more cumbersome and we refer to Refs. [56-59]. After calculating the contributions of potential and kinetic energies, the total energy per particle is written as

$$
\langle H\rangle=\frac{n}{2} \int d \mathbf{r} g(r)\left[v(r)-\frac{\hbar^{2}}{2 m} \nabla^{2} \ln f(r)\right] .
$$

As we can see in this expression, the energy per particle is related to $g(r)$ and $f(r)$. It is convenient to do the variation with respect to $g(r)$ instead of $f(r)$. The simplest way to do this is done within the HNC/0 approximation, where we can express $f(r)$ as

$$
\ln f(r)=\frac{1}{2}\left[\ln g(r)-\frac{1}{(2 \pi)^{3} n} \int \frac{(S(q)-1)^{2}}{S(q)} e^{i \mathbf{q} \cdot \mathbf{r}} d \mathbf{q}\right] .
$$

After inserting Eq. (A9) into Eq. (A8), the energy per particle is obtained in terms of $g(r)$ :

$$
\begin{aligned}
\langle H\rangle= & \frac{n}{2} \int d \mathbf{r} g(r) v(r)-\frac{\hbar^{2}}{8 m} \frac{1}{(2 \pi)^{3} n} \\
& \times \int d \mathbf{q} q^{2} \frac{[S(q)-1]^{3}}{S(q)}-\frac{n}{2} \frac{\hbar^{2}}{4 m} \int d \mathbf{r} g(r) \nabla^{2} \ln g(r) .
\end{aligned}
$$

To obtain equations for the optimal pair distribution function $g(r)$, we minimize the energy expectation value of the system with respect to $g(r)$ by performing a functional derivative

$$
\frac{\partial\langle H\rangle}{\partial \sqrt{g(r)}}=0,
$$

and what ensues is Eq. (2).

\section{APPENDIX B: COMPARISON BETWEEN KALLIO-PIILO AND LADDER+APPROXIMATIONS FOR THE EFFECTIVE INTERACTION}

Recently, Panholzer, Hobbiger, and Böhm (PHB) proposed a new particle-hole effective potential for the Fermihypernetted-chain approximation, based on the approximate summation of ladder and ring diagrams [47]. The selfconsistent equations of PHB essentially become identical to
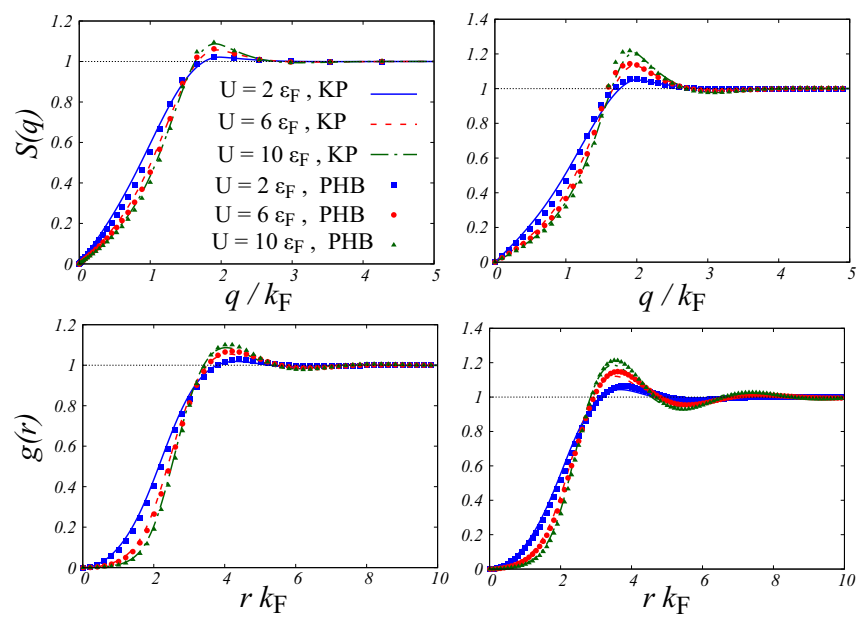

FIG. 8. Comparison between the results of KP (lines) and PHB (symbols) approximations for the Fermi potentials of FHNC method, for static structure factor (top) and pair distribution function (bottom) of 3D (left) and 2D (right) systems of Rydberg dressed fermions for several coupling strengths. The dimensionless soft-core radius is $r_{c}=2$ in all panels.

the KP equations if one replaces the Fermi potential $w_{\mathrm{F}}(r)$ in equation (5) with

$$
w_{\mathrm{F}}^{\mathrm{PHB}}(r)=\frac{\hbar^{2}}{m} \frac{\nabla_{\mathrm{r}}^{2} \sqrt{g_{0}(r)}}{\sqrt{g_{0}(r)}}+w_{l}(r)
$$

where

$$
w_{l}(q)=\frac{\varepsilon_{q}}{2 n}\left[2 S(q)\left(1-\frac{1}{S_{0}^{3}(q)}\right)-3\left(1-\frac{1}{S_{0}^{2}(q)}\right)\right] .
$$

In Fig. 8, we compare the results for the static structure factor and pair distribution function of Rydberg-dressed fermions, obtained from the KP and PHB approximations. Both methods give very similar results for the set of parameters we have checked here. The PHB method gives slightly more pronounced peaks for both the static structure factor and the pair distribution function at strong couplings. Validation of both methods and their performance at different coupling strengths requires more accurate results, e.g., those obtained from the quantum Monte Carlo simulations. Such benchmark data are not yet available in the literature.
[1] H. Bernien, S. Schwartz, A. Keesling, H. Levine, A. Omran, H. Pichler, S. Choi, A. S. Zibrov, M. Endres, M. Greiner, V. Vuletić, and M. D. Lukin, Nature (London) 551, 579 (2017).

[2] J. Zeiher, R. Van Bijnen, P. Schauß, S. Hild, J.-y. Choi, T. Pohl, I. Bloch, and C. Gross, Nat. Phys. 12, 1095 (2016).

[3] H. Labuhn, D. Barredo, S. Ravets, S. de Léséleuc, T. Macrì, T. Lahaye, and A. Browaeys, Nature (London) 534, 667 (2016).
[4] J. B. Balewski, A. T. Krupp, A. Gaj, S. Hofferberth, R. Löw, and T. Pfau, New J. Phys. 16, 063012 (2014).

[5] O. Firstenberg, C. S. Adams, and S. Hofferberth, J. Phys. B: At., Mol. Opt. Phys. 49, 152003 (2016).

[6] Y. Dudin and A. Kuzmich, Science 336, 887 (2012).

[7] C. S. Adams, J. D. Pritchard, and J. P. Shaffer, J. Phys. B: At., Mol. Opt. Phys. 53, 012002 (2019).

[8] M. Saffman, T. G. Walker, and K. Mølmer, Rev. Mod. Phys. 82, 2313 (2010). 
[9] L. Isenhower, E. Urban, X. L. Zhang, A. T. Gill, T. Henage, T. A. Johnson, T. G. Walker, and M. Saffman, Phys. Rev. Lett. 104, 010503 (2010).

[10] M. D. Lukin, M. Fleischhauer, R. Cote, L. M. Duan, D. Jaksch, J. I. Cirac, and P. Zoller, Phys. Rev. Lett. 87, 037901 (2001).

[11] A. Browaeys and T. Lahaye, Interacting cold rydberg atoms: A toy many-body system, in Niels Bohr, Progress in Mathematical Physics, edited by O. Darrigol, B. Duplantier, J. M. Raimond, and V. Rivasseau, Vol. 68 (Birkhäuser, Cham, 2016), pp. 177-198.

[12] H. Weimer, M. Müller, I. Lesanovsky, P. Zoller, and H. P. Büchler, Nat. Phys. 6, 382 (2010).

[13] M. Płodzień, T. Sowiński, and S. Kokkelmans, Sci. Rep. 8, 9247 (2018).

[14] T. Karpiuk, M. Brewczyk, K. Rzażewski, A. Gaj, J. B. Balewski, A. T. Krupp, M. Schlagmüller, R. Löw, S. Hofferberth, and T. Pfau, New J. Phys. 17, 053046 (2015).

[15] N. Henkel, R. Nath, and T. Pohl, Phys. Rev. Lett. 104, 195302 (2010).

[16] A. Browaeys, D. Barredo, and T. Lahaye, J. Phys. B: At., Mol. Opt. Phys. 49, 152001 (2016).

[17] Y.-Y. Jau, A. Hankin, T. Keating, I. Deutsch, and G. Biedermann, Nat. Phys. 12, 71 (2016).

[18] J. Zeiher, J.-y. Choi, A. Rubio-Abadal, T. Pohl, R. van Bijnen, I. Bloch, and C. Gross, Phys. Rev. X 7, 041063 (2017).

[19] S. Hollerith, J. Zeiher, J. Rui, A. Rubio-Abadal, V. Walther, T. Pohl, D. M. Stamper-Kurn, I. Bloch, and C. Gross, Science 364, 664 (2019).

[20] G. Pupillo, A. Micheli, M. Boninsegni, I. Lesanovsky, and P. Zoller, Phys. Rev. Lett. 104, 223002 (2010).

[21] M. Boninsegni and N. V. Prokof'ev, Rev. Mod. Phys. 84, 759 (2012).

[22] F. Cinti, P. Jain, M. Boninsegni, A. Micheli, P. Zoller, and G. Pupillo, Phys. Rev. Lett. 105, 135301 (2010).

[23] S. Prestipino, A. Sergi, and E. Bruno, Phys. Rev. B 98, 104104 (2018).

[24] F. Cinti, T. Macrí, W. Lechner, G. Pupillo, and T. Pohl, Nat. Commun. 5, 3235 (2014).

[25] G. Semeghini, G. Ferioli, L. Masi, C. Mazzinghi, L. Wolswijk, F. Minardi, M. Modugno, G. Modugno, M. Inguscio, and M. Fattori, Phys. Rev. Lett. 120, 235301 (2018).

[26] I. Ferrier-Barbut, H. Kadau, M. Schmitt, M. Wenzel, and T. Pfau, Phys. Rev. Lett. 116, 215301 (2016).

[27] C. Cabrera, L. Tanzi, J. Sanz, B. Naylor, P. Thomas, P. Cheiney, and L. Tarruell, Science 359, 301 (2018).

[28] I. Seydi, S. H. Abedinpour, R. E. Zillich, R. Asgari, and B. Tanatar, Phys. Rev. A 101, 013628 (2020).

[29] M. Schmitt, M. Wenzel, F. Böttcher, I. Ferrier-Barbut, and T. Pfau, Nature (London) 539, 259 (2016).

[30] M. Guo and T. Pfau, Front. Phys. 16, 32202 (2021).

[31] T. E. Lee, S. Gopalakrishnan, and M. D. Lukin, Phys. Rev. Lett. 110, 257204 (2013).

[32] B. Xiong, H. H. Jen, and D.-W. Wang, Phys. Rev. A 90, 013631 (2014).

[33] X. Li and S. D. Sarma, Nat. Commun. 6, 7137 (2015).
[34] A. W. Glaetzle, M. Dalmonte, R. Nath, I. Rousochatzakis, R. Moessner, and P. Zoller, Phys. Rev. X 4, 041037 (2014).

[35] L. Tanzi, E. Lucioni, F. Famà, J. Catani, A. Fioretti, C. Gabbanini, R. N. Bisset, L. Santos, and G. Modugno, Phys. Rev. Lett. 122, 130405 (2019).

[36] F. Böttcher, J.-N. Schmidt, M. Wenzel, J. Hertkorn, M. Guo, T. Langen, and T. Pfau, Phys. Rev. X 9, 011051 (2019).

[37] L. Chomaz, D. Petter, P. Ilzhöfer, G. Natale, A. Trautmann, C. Politi, G. Durastante, R. M. W. van Bijnen, A. Patscheider, M. Sohmen, M. J. Mark, and F. Ferlaino, Phys. Rev. X 9, 021012 (2019).

[38] W.-H. Li, T.-C. Hsieh, C.-Y. Mou, and D.-W. Wang, Phys. Rev. Lett. 117, 035301 (2016).

[39] R. Khasseh, S. H. Abedinpour, and B. Tanatar, Phys. Rev. A 96, 053611 (2017).

[40] A. Keleş, E. Zhao, and X. Li, Phys. Rev. A 101, 023624 (2020).

[41] B. Davoudi, R. Asgari, M. Polini, and M. P. Tosi, Phys. Rev. B 68, 155112 (2003).

[42] R. Asgari, B. Davoudi, and M. Tosi, Solid State Commun. 131, 301 (2004).

[43] S. H. Abedinpour, R. Asgari, B. Tanatar, and M. Polini, Ann. Phys. (NY) 340, 25 (2014).

[44] H. M. Böhm, R. Holler, E. Krotscheck, and M. Panholzer, Phys. Rev. B 82, 224505 (2010).

[45] E. Krotscheck, J. Low Temp. Phys. 119, 103 (2000).

[46] A. Kallio and J. Piilo, Phys. Rev. Lett. 77, 4237 (1996).

[47] M. Panholzer, R. Hobbiger, and H. Böhm, Phys. Rev. B 99, 195156 (2019).

[48] G. F. Giuliani and G. Vignale, Quantum Theory of the Electron Liquid (Cambridge University Press, Cambridge, 2005).

[49] I. Seydi, S. H. Abedinpour, R. Asgari, and B. Tanatar, Phys. Rev. A 98, 063623 (2018).

[50] R. Asgari and B. Tanatar, Phys. Rev. B 74, 075301 (2006).

[51] I. S. Gradshteyn and I. M. Ryzhik, Table of Integrals, Series, and Products (Elsevier/Academic Press, Amsterdam, 2007).

[52] K. Singer, J. Stanojevic, M. Weidemüller, and R. Côté, J. Phys. B: At., Mol. Opt. Phys. 38, S295 (2005).

[53] K. S. Singwi, M. P. Tosi, R. H. Land, and A. Sjölander, Phys. Rev. 176, 589 (1968).

[54] I. Seydi, Ph.D. thesis, Institute for Advanced Studies in Basic Sciences (IASBS), Zanjan, Iran, 2019 (unpublished).

[55] J.-P. Hansen and I. R. McDonald, Theory of Simple Liquids: With Applications to Soft Matter (Elsevier/Academic Press, Oxford, 2013).

[56] R. F. Bishop, in Microscopic Quantum Many-Body Theories and Their Applications, Lecture Notes in Physics, Vol. 510, edited by J. Navarro and A. Polls (Springer-Verlag, Berlin, 1998), pp. $1-70$.

[57] A. Fabrocini, S. Fantoni, and E. Krotscheck, Introduction to Modern Methods of Quantum Many-body Theory and Their Applications, Series on Advances in Quantum Many-Body Theory, Vol. 7 (World Scientific, River Edge, NJ, 2002).

[58] E. Krotscheck, Phys. Rev. B 33, 3158 (1986).

[59] C. C. Chang and C. E. Campbell, Phys. Rev. B 13, 3779 (1976). 